\title{
Single top quark production cross-section measurements using the ATLAS and CMS detectors at the LHC
}

\author{
Kevin Finelli* \\ University of Sydney (AU) \\ On behalf of the ATLAS and CMS collaborations \\ E-mail: kevin.finelli@cern.ch
}

\begin{abstract}
Measurements of single top quark production in proton-proton collisions at 7 and $8 \mathrm{TeV}$ using the ATLAS and CMS detectors at the LHC are presented. For the $t$-channel process, the single top quark and single anti-top total production cross-section ratio, as well as a measurement of the inclusive and fiducial production cross-sections are presented. In addition, a measurement of the production cross-section of a single top quark in association with a $W$ boson is presented. All measurements are compared to state-of-the-art theoretical calculations and the CKM matrix element $\left|V_{t b}\right|$ is determined. The $s$-channel production is also explored and limits on exotic production in single top quark processes are discussed. This includes the search for additional $W$ bosons and a search for monotop final states.
\end{abstract}

XXIII International Workshop on Deep-Inelastic Scattering,

27 April - May 12015

Dallas, Texas

${ }^{*}$ Speaker. 


\section{Introduction}

The production of single top quarks through electroweak interactions provides a channel with rich phenomenology to study the top quark. The top quark decays before it hadronizes, providing physicists with the only opportunity to study partons without hadronization effects interfering. The relatively high mass of the top quark (approximately $173 \mathrm{GeV}$ ) makes it theoretically interesting and important for corrections to the Higgs mass, hierarchy problems, and electroweak symmetry breaking.

The production of single top quarks provides a way to test the standard model that is distinct from strong top pair production and is accessible to the ATLAS [1] and CMS [2] experiments. For example, the CKM matrix element $V_{t b}$ can be probed directly, and further sensitivity to the effects of parton distribution functions (PDF) is available. Furthermore, final states with the production of a single top can provide a powerful probe for new physics beyond the standard model. Examples include tests for anomalous $W t b$ vertex couplings, flavor changing neutral currents, and the production of a heavy charged $W^{\prime}$ boson.

There are three leading order diagrams of single top production at the LHC. Of the three, the $t$-channel has the highest production cross-section, and its final state is characterized by a $b$-quarkinitiated jet from the decay of the top quark, a forward light-flavor jet from the spectator quark, and an isolated charged lepton with missing transverse momentum from the decay of the $W$ coming from the top quark. The $s$-channel has the smallest production cross-section at the LHC owing to its quark-anti-quark initial state, making it a difficult signal to observe. Its signature is two $b$-quark-initiated jets, and an isolated charged lepton with missing transverse momentum from the decay of the $W$ coming from the top quark. The $W t$ associated production channel has a detector signature that is very similar to top quark pair production. It is typically identified with one $b$ quark-initiated jet, two isolated charged leptons, and missing transverse momentum from the two $W$ bosons produced in the event.

Several analyses further utilize single top final states to measure properties of the $W t b$ vertex. Anomalous $W t b$ couplings are parameterized by the following effective Lagrangian:

$$
\mathscr{L}_{W t b}=-\frac{g}{\sqrt{2}} \bar{b} \gamma^{\mu}\left(V_{\mathrm{L}} P_{\mathrm{L}}+V_{\mathrm{R}} P_{\mathrm{R}}\right) t W_{\mu}^{-}-\frac{g}{\sqrt{2}} \bar{b} \frac{i \sigma^{\mu v} q_{v}}{m_{W}}\left(g_{\mathrm{L}} P_{\mathrm{L}}+g_{\mathrm{R}} P_{\mathrm{R}}\right) t W_{\mu}^{-}+\text {h.c. }
$$

where the $V_{\mathrm{L}}$ and $V_{\mathrm{R}}$ are the left- and right-handed vector couplings, and $g_{\mathrm{L}}$ and $g_{\mathrm{R}}$ are the left- and right-handed tensor couplings. In the standard model, $V_{\mathrm{L}}=V_{t b} \approx 1$, and the other three couplings are zero. Any deviation from these values is evidence for new physics in the $W t b$ vertex.

\section{Production cross-sections}

ATLAS [3] and CMS [4] have performed measurements of the $t$-channel single top production cross-section using $\sqrt{s}=8 \mathrm{TeV}$ data. In these analyses, exactly two jets are selected: one is required to be $b$-tagged and central to the detector, the other must be untagged and may be central or forward. A discriminant is constructed to separate signal from background; ATLAS uses a neural network discriminant, and CMS uses the $|\eta|$ distribution of the untagged jet.

The measurements are systematics-limited, with the main systematic uncertainties being those related to the jet energy scale (JES) and Monte Carlo (MC) modelling of the signal process. The 
ATLAS measurement produces full and fiducial cross-sections; the fiducial region is defined at the level of MC particles to be the same as the selection on reconstructed-level objects in order to minimize modelling uncertainty. The ATLAS fiducial results are summarized in Figure 1. Also shown in Figure 1 are the CMS measurements of $R_{t-c h}$., the ratio of top to anti-top quark production, which provides additional discriminating power to test QCD predictions interfaced to a variety of PDF sets.
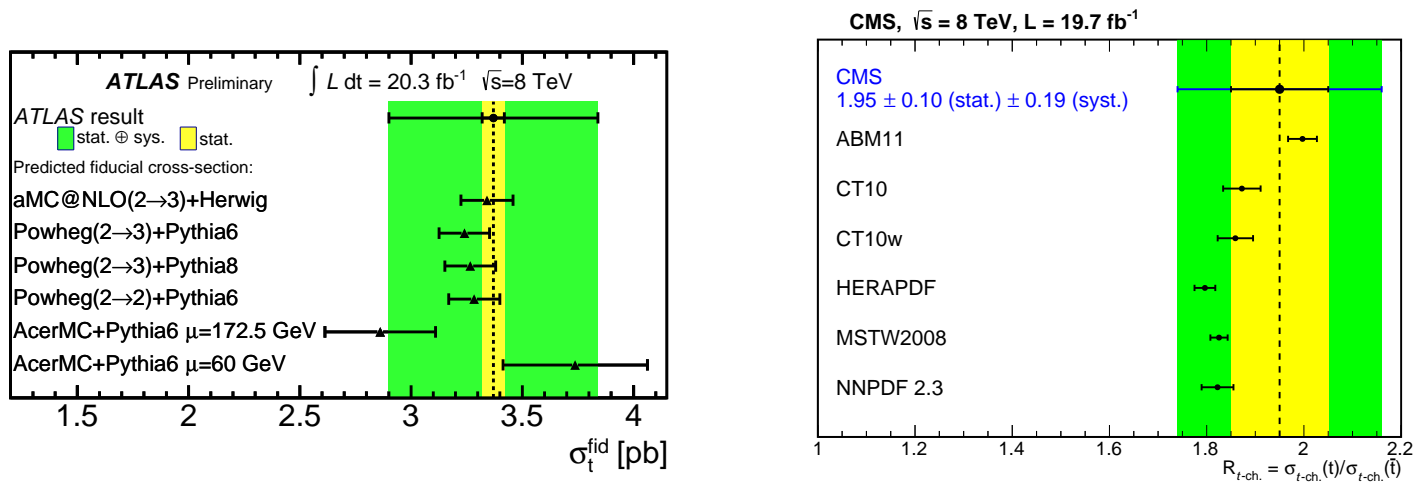

Figure 1: Results of $t$-channel cross-section measurements from ATLAS [3] and CMS [4].

Searches for single top production in the $s$-channel were performed by ATLAS [5] and CMS [6] using data with $\sqrt{s}=8 \mathrm{TeV}$. The analyses from both experiments select events with large missing transverse momentum, one isolated lepton and exactly two $b$-tagged jets. The selected events are separated using a boosted decision tree (BDT) discriminant, and the $s$-channel signal is extracted from a fit to this discriminant. The observed cross-section upper limits (ATLAS: observed $14.6 \mathrm{pb}$, expected $15.7 \mathrm{pb}$, CMS: observed $11.5 \mathrm{pb}$, expected $17.0 \mathrm{pb}$ ) are consistent with the most precise available theoretical calculations [7].

Analyses of single top production in association with a $W$ boson are performed by ATLAS [8] and CMS [9] with $\sqrt{s}=8 \mathrm{TeV}$ data, and a statistical combination of the results is available [10]. Events with a pair of opposite-signed leptons and exactly one $b$-tagged jet are selected, and a BDT (shown in Figure 2) is constructed to discriminate the $W t$ signal against the much larger top quark pair background. The production cross-sections and the value of $\left|V_{t b}\right|$ are extracted from a fit to the BDT discriminant. The greatest significance to reject the background-only hypothesis comes from CMS and is observed to be $6.1 \sigma$ (5.4 $\sigma$ expected).

A summary of the $t$-channel, $s$-channel, and $W t$ inclusive cross-section measurements and searches discussed here is presented in the summary plot in Figure 3.

\section{Properties of the $W t b$ vertex}

ATLAS [12] and CMS [13] perform analyses probing the $W t b$ vertex for anomalous couplings. In the SM, the $W t b$ vertex is described by a left-handed vector coupling, but right-handed or tensor couplings are predicted by certain new physics scenarios. The ATLAS analysis is designed to look for a CP-violating imaginary component of the right-handed tensor coupling $g_{R}$, which is extracted 

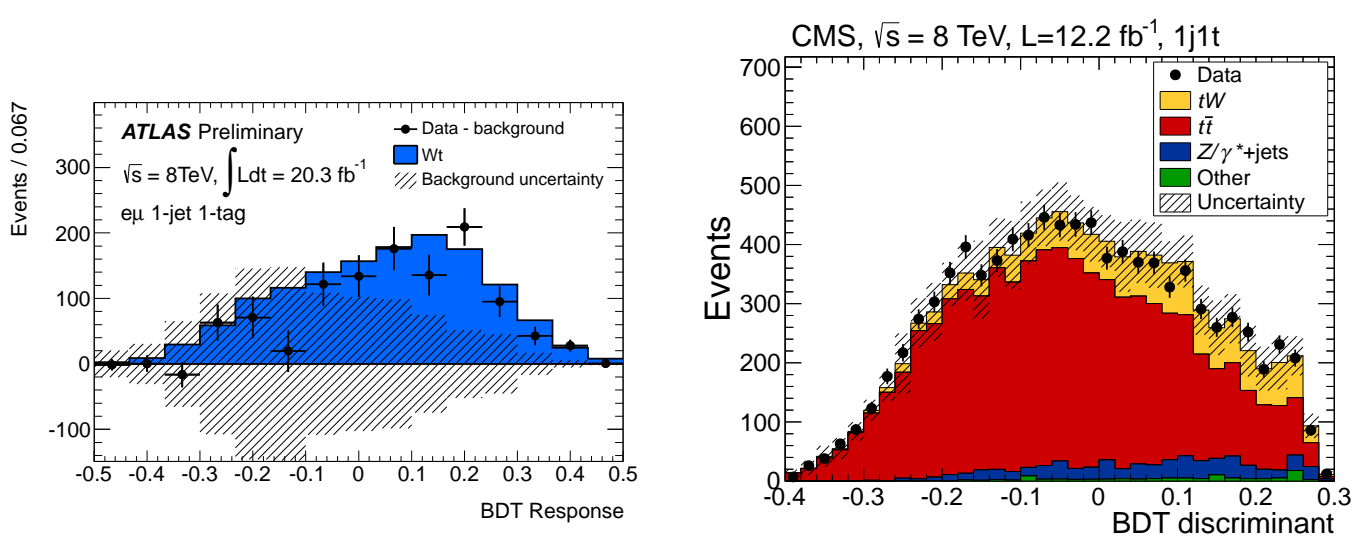

Figure 2: Discriminants used to separate signal from background within the signal region of the ATLAS [5] (left) and CMS [6] (right) Wt inclusive cross-section measurements.

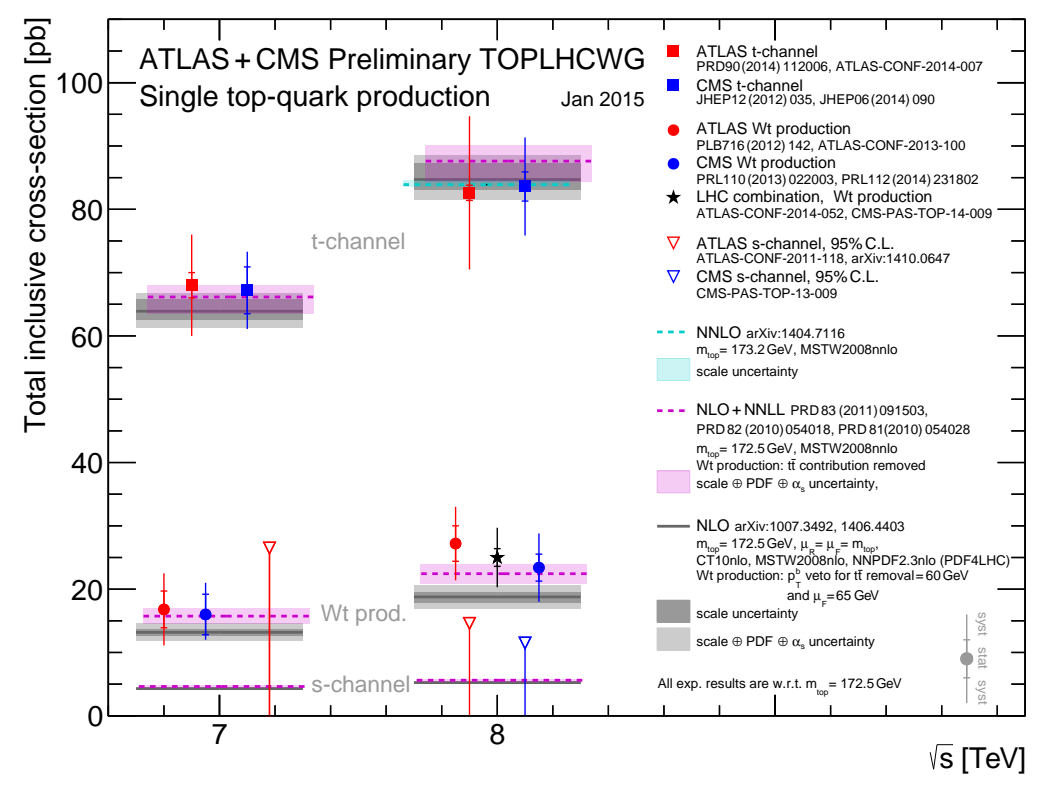

Figure 3: Summary of inclusive cross-section measurements and theoretical predictions in the $t$-channel, $s$-channel, and $W t$ associated production channel [11].

from angular asymmetry measurements in the $t$-channel. CMS searches for anomalous values of the left- and right-handed vector and left-handed tensor couplings using a series of Bayesian neural networks trained to discriminate these anomalous coupling signals in $t$-channel single top events. Figure 4 shows the results of the measurements: the allowed value of $\operatorname{Im}\left(g_{R}\right)$ from ATLAS and two-dimensional exclusion limits on pairs of coupling strengths from CMS.

A measurement of the $W$ boson helicity is performed using $\sqrt{s}=8 \mathrm{TeV}$ data by CMS [14] using $t$-channel single top events. The helicity fractions $F_{\mathrm{L}}, F_{0}$, and $F_{\mathrm{R}}$ are measured to a similar precision to that achieved in $t \bar{t}$ events, and found to be in good agreement with SM NNLO pre- 
dictions. The helicity measurements are also interpreted to set limits on the real part of the Wtb anomalous couplings, $g_{\mathrm{L}}$ and $g_{\mathrm{R}}$. The helicity fractions and anomalous coupling limits are shown in Figure 5.
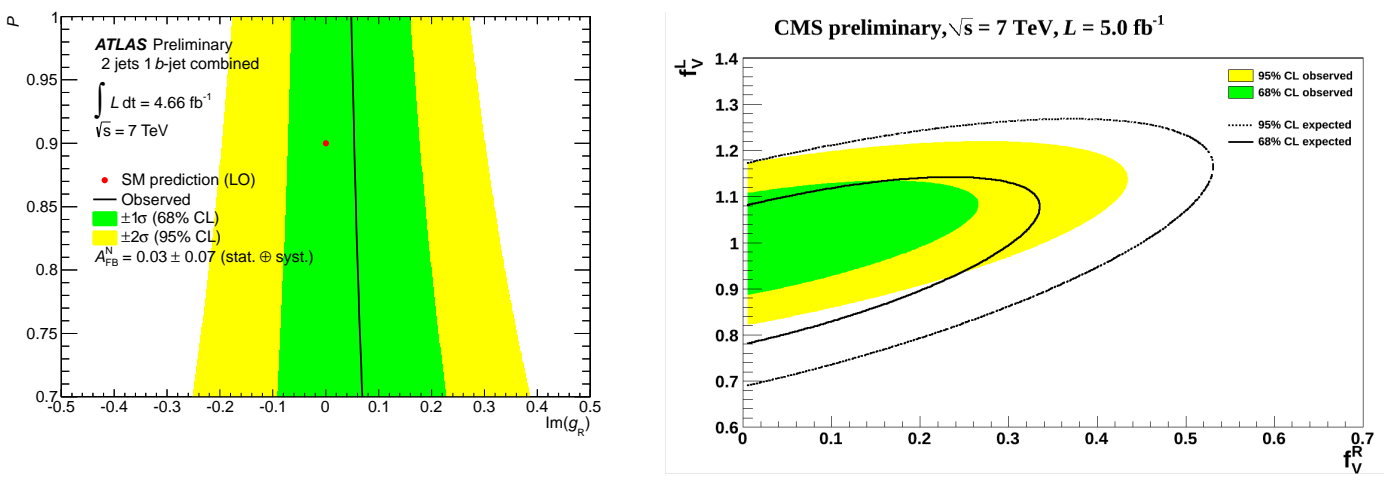

Figure 4: Constraints in the top quark polarization versus imaginary component of the right-handed $W t b$ tensor coupling from the ATLAS analysis [12] (left). Exclusion limits in two-dimensions on left- and righthanded vector $W t b$ coulings for the observed and expected limits from the CMS analysis [13] (right).
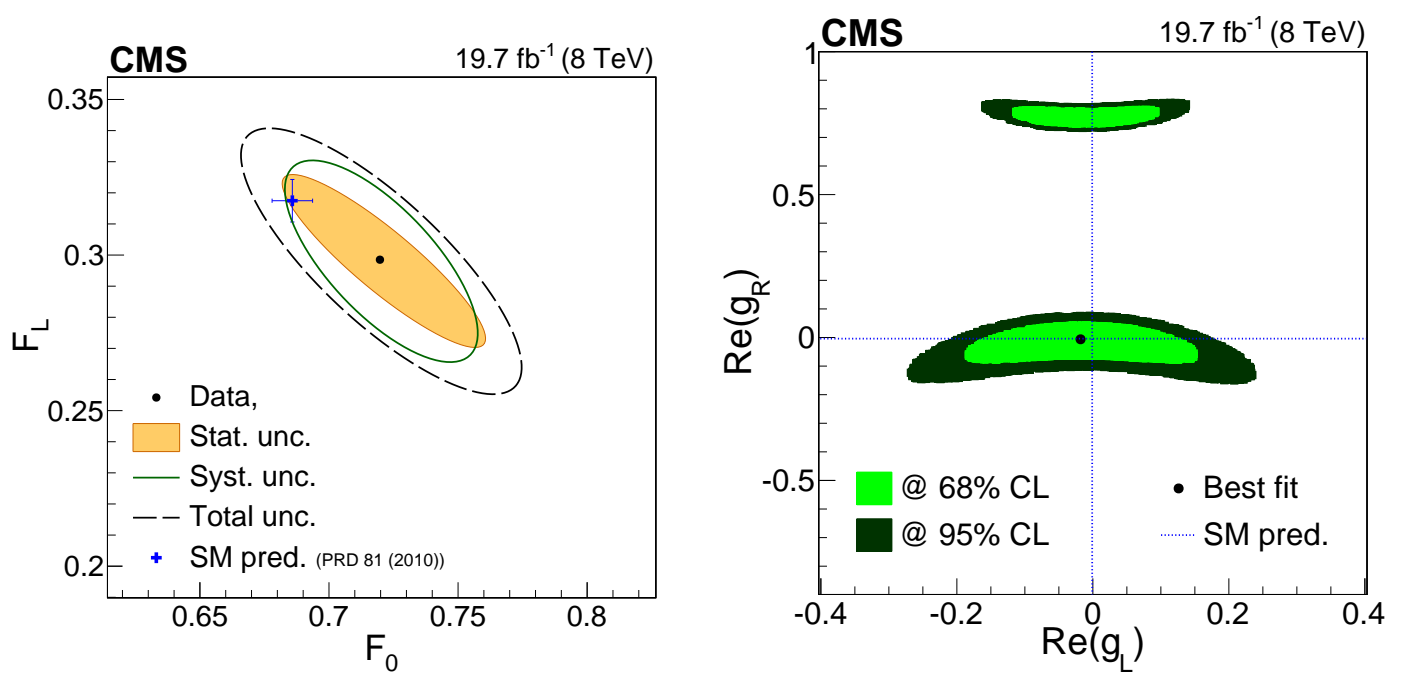

Figure 5: Results of the CMS $W$ helicity measurement [14]: left-handed and longitudinal $W$ boson helicity fractions (left) and exclusion limits on the real part of the $g_{\mathrm{L}}$ and $g_{\mathrm{R}}$ anomalous couplings, with $V_{\mathrm{L}}=1$ and $V_{\mathrm{R}}=0$ (right).

A measurement of the polarization of the top quark in $t$-channel events is performed by CMS [15]. Events are unfolded to obtain the parton-level distribution of a spin-analyzing angle sensitive to the polarization of the top quark. The asymmetry of this angle is used to extract the polarization of the top quark, which is found to be $P_{t}=0.83 \pm 0.12$ (stat.) \pm 0.32 (syst.) under the assumption that the spin analyzing power of the charged lepton is $100 \%$. (The spin analyzing power of the lepton may be modified by anomalous top quark couplings in the Wtb vertex.) 


\section{Searches for new physics}

ATLAS [16] and CMS [17] have performed searches for heavy $W^{\prime}$ resonances decaying to $t b$ using $\sqrt{s}=8 \mathrm{TeV}$ data. Events with two $b$-tagged jets, one charged lepton, and light-flavor jets are selected, and signal templates are fitted to the distribution of $m_{t b}$ (CMS) or the output of a BDT (ATLAS). No excess is observed and limits on left- and right-handed $W^{\prime}$ boson masses are set. The mass limits on right-handed coupling $W^{\prime}$ bosons are shown in Figure 6.
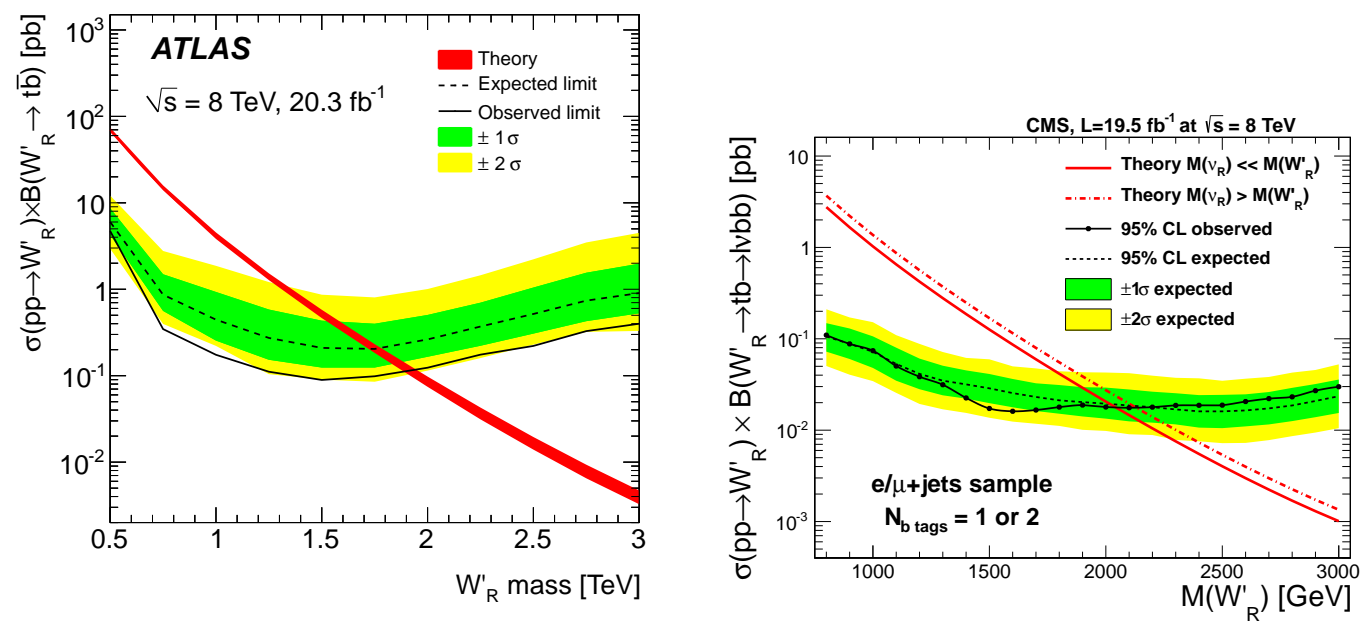

Figure 6: Observed and expected 95\% CL limits on the $W^{\prime}$ boson cross-section times branching ratio as a function of the boson mass for right-handed $W^{\prime}$ bosons from ATLAS [16] (left) and CMS [17] (right).

ATLAS [18] and CMS [19] have performed searches at $\sqrt{s}=8 \mathrm{TeV}$ for events with invisible particles produced in association with single top quarks, also known as monotop events. ATLAS selects leptonically decaying top quarks and uses a cut-and-count analysis to study resonant and non-resonant models of dark matter production. CMS selects hadronically decaying top quarks and investigates scalar and vector dark matter models using a likelihood fit to test for the presence of signal. No excesses are observed in either analysis and limits are placed on the various models of dark matter production. Figure 7 shows the observed and expected limits on the cross-section times branching ratio for the non-resonant and vector dark matter models studied by ATLAS and CMS.

\section{Conclusion}

Measurements of single top quark production in proton-proton collisions at 7 and $8 \mathrm{TeV}$ from the ATLAS and CMS experiments are presented. Inclusive cross-section measurements are performed in the $t$-channel, and searches are performed in the $s$-channel and for $W t$ production, with a combined $6.1 \sigma$ discovery reported for $W t$ production. Properties of the $W t b$ vertex accessible in $t$-channel single top events are measured. Limits on exotic production in single top quark processes are also investigated, including the search for $W$ bosons and a search for monotop final states. 

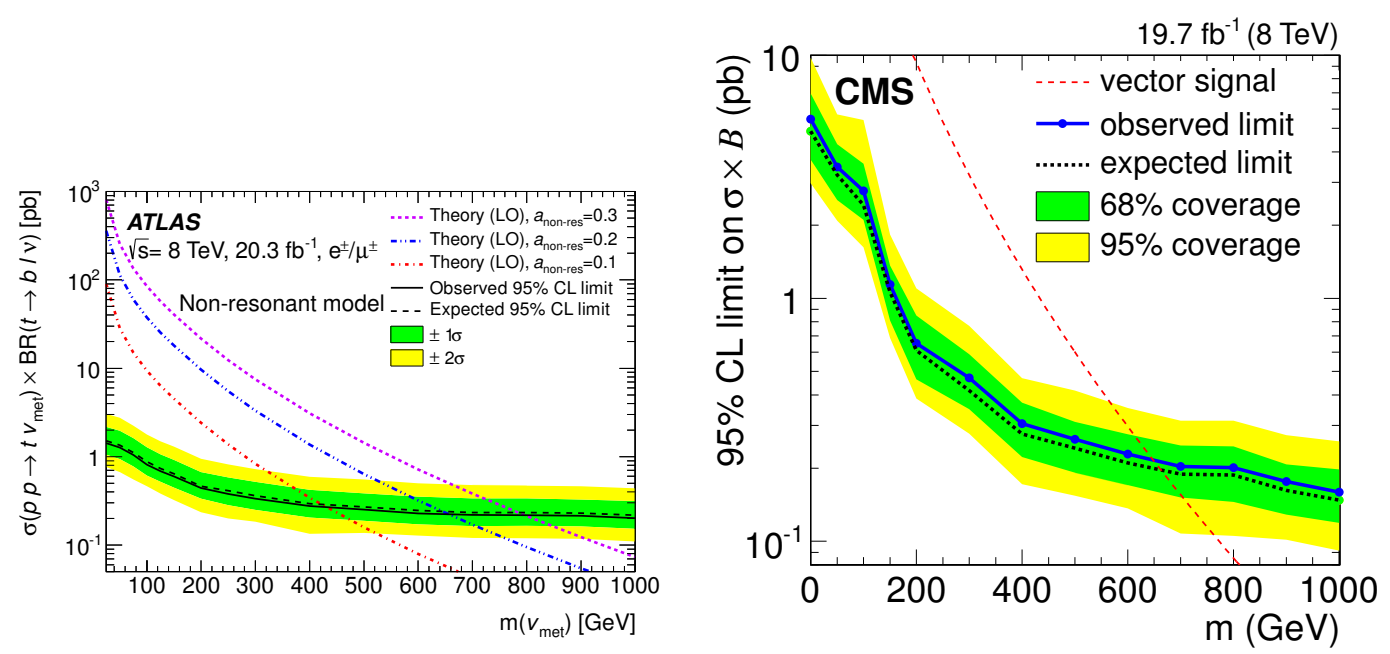

Figure 7: Observed and expected limits on the cross-section times branching ratio for non-resonant dark matter production from ATLAS [18] (left) and for vector dark matter production from CMS [19].

\section{References}

[1] ATLAS Collaboration, 2008 JINST 3 S08003.

[2] CMS Collaboration, 2008 JINST 3 S08004.

[3] ATLAS Collaboration, ATLAS-CONF-2014-007.

[4] CMS Collaboration, JHEP 06 (2014) 090.

[5] ATLAS Collaboration, Phys. Lett. B740 (2015) 118.

[6] CMS Collaboration, CMS PAS TOP-13-009.

[7] N. Kidonakis, Phys. Rev. D 83 (2011) 091503.

[8] ATLAS Collaboration, ATLAS-CONF-2013-100.

[9] CMS Collaboration, Phys. Rev. Lett. 112 (2014) 231802.

[10] ATLAS and CMS Collaborations, ATLAS-CONF-2014-052, CMS PAS TOP-14-009.

[11] TOPLHC WG Summary Plots, accessed 23 June 2015, <https://twiki.cern.ch/twiki/bin/view/LHCPhysics/TopLHCWGSummaryPlots>.

[12] ATLAS Collaboration, ATLAS-CONF-2013-032.

[13] CMS Collaboration, CMS PAS TOP-14-007.

[14] CMS Collaboration, JHEP 01 (2015) 053.

[15] CMS Collaboration, CMS PAS TOP-13-001.

[16] ATLAS Collaboration, Physics Letters B 743 (2015) 235-255.

[17] CMS Collaboration, JHEP 05 (2014) 108.

[18] ATLAS Collaboration, Eur. Phys. J. C (2015) 75:79.

[19] CMS Collaboration, Phys. Rev. Lett. 114 (2015) 101801. 